\title{
Evolutions in 3D numerical relativity using fixed mesh refinement
}

\author{
Erik Schnetter, ${ }^{1}$ Scott H. Hawley, ${ }^{2}$ and Ian Hawke ${ }^{3}$ \\ ${ }^{1}$ Institut für Astronomie und Astrophysik, Universität Tübingen, Auf der Morgenstelle, D-72076 Tübingen, Germany \\ ${ }^{2}$ Center for Relativity, University of Texas at Austin, Austin, TX 78712, USA I0 \\ ${ }^{3}$ Max-Planck-Institut für Gravitationsphysik, Albert-Einstein-Institut, D-14476 Golm, Germany
}

\begin{abstract}
We present results of 3D numerical simulations using a finite difference code featuring fixed mesh refinement (FMR), in which a subset of the computational domain is refined in space and time. We apply this code to a series of test cases including a robust stability test, a nonlinear gauge wave and an excised Schwarzschild black hole in an evolving gauge. We find that the mesh refinement results are comparable in accuracy, stability and convergence to unigrid simulations with the same effective resolution. At the same time, the use of FMR reduces the computational resources needed to obtain a given accuracy. Particular care must be taken at the interfaces between coarse and fine grids to avoid a loss of convergence at higher resolutions, and we introduce the use of "buffer zones" as one resolution of this issue. We also introduce a new method for initial data generation, which enables higher-order interpolation in time even from the initial time slice. This FMR system, "Carpet", is a driver module in the freely available Cactus computational infrastructure, and is able to endow generic existing Cactus simulation modules ("thorns") with FMR with little or no extra effort.
\end{abstract}

PACS numbers: $04.25 . \mathrm{Dm}$ 04.20.-q 04.70.Bw

\section{INTRODUCTION}

Currently many researchers are involved in efforts to predict the gravitational waveforms emitted by the inspiral and merger of compact binaries. The direct numerical simulation of these binary sources has been regarded as the best, if not only, way to obtain information about the waves emitted during the merger event itself. Many numerical simulation methods are currently in use, and among the most popular of these is the use of finite difference approximations to Einstein's equations on a uniform mesh or grid.

It is often the case in simulations of physical systems that the most interesting phenomena may occur in only a subset of the computational domain. In the other regions of the domain it may be possible to use a less accurate approximation, thereby reducing the computational resources required, and still obtain results which are essentially similar to those obtained if no such reduction is made. In particular, we may consider using a computational mesh which is non-uniform in space and time, using a finer mesh resolution in the "interesting" regions where we expect it to be necessary, and using a coarser resolution in other areas. This is what we mean by mesh refinement (MR). In the case of large-scale simulations, where memory and computing time are at a premium, the effective use of MR can allow for simulations achieving effective (fine-grid) resolutions which would be, on a practical level, impossible otherwise. This also implies that some systems requiring a large dynamic range of resolution may only be possible to simulate using mesh refinement. One hopes that the use of fewer total grid points in an efficient mesh refinement application, as opposed to a uniform, single grid or unigrid application, may result in a saving of computing time required to arrive at a solution. It is always the case, however, that the reduction of memory requirements afforded by mesh refinement, especially in 3D, could allow for highly accurate simulations which simply may not fit in the memory of a given computer in a unigrid setting.

In many simulations it may be desirable for the computational mesh to adaptively refine in response to some criterion such as the local truncation error. Such adaptive mesh refinement (AMR) systems provide, ideally, a very high degree of computational efficiency for a given problem. AMR schemes have been in use for decades [1], particularly in the fluid dynamics community [2, 3]. Applications to to astrophysical systems have been underway for quite some time (e.g., [4, 5, 6, 7]]). In numerical relativity, Choptuik [8, 9] introduced many researchers to AMR through his results using a 1D code for studying critical phenomena. Since the early 90 's efforts have been underway in the numerical relativity community to develop and employ AMR applications in two and three dimensions, in studies of waves [10, 11, 12, 13, 14, 15], critical collapse [9, 16, 17, 18], the initial data problem [7, 19, 20], inhomogeneous cosmologies

\footnotetext{
*Max-Planck-Institut für Gravitationsphysik, Albert-Einstein-Institut, D-14476 Golm, Germany; Electronic address: schnetter@aei.mpg.de

$\dagger$ Electronic address: shawley@physics.utexas.edu

‡Electronic address: hawke@aei.mpg.de
} 
[21], Schwarzschild black holes [12, 22, 23], characteristic methods [24], and a binary black hole system [25]. In several of these cases, the use of mesh refinement provided not only a more efficient means of computing a solution, but even allowed the authors to obtain solutions which would not have been possible via standard unigrid methods, given existing computing technology at the time.

One step in the development of an AMR code is the ability to handle regions of different resolution which are known in advance - fixed mesh refinement (FMR). Thus we view our present work as in some sense a precursor to full 3D AMR simulations. However, the FMR code may be sufficient in its own right, as full AMR may not be necessary for problems where the region of interest is well known beforehand.

An important point should be made regarding the benefits of mesh refinement for reducing storage requirements (and computational costs). It is well known that pseudospectral collocation methods offer exponential convergence as the number of collocation points is increased (for sufficiently smooth functions, which are present in many systems of interest to numerical relativists), and such methods are able to yield extremely high accuracies via simulations which require storage afforded by a typical desktop computer [26, 27, 28, 29, 30]. Since FMR makes use of finite difference methods, we can obtain at best only polynomial convergence as we increase the resolution of all grids. Our choice of finite difference methods and FMR are based on a choice of physical systems of interest, and on an observation.

One reason we are motivated to use mesh refinement is that we are interested in systems which are "non-smooth" by the standards of pseudospectral methods: hydrodynamics, and gravitational collapse. In hydrodynamics, the formation of discontinuities - shocks - from smooth initial data is a generic phenomena. In gravitational collapse, features may appear on smaller and smaller spatial scales, requiring a means of resolving these features as they appear, either via a truly adaptive algorithm (in which, e.g., the truncation error is used as a refinement criterion [1]) or a "progressive" mesh refinement system in which nested grids are "turned on" at smaller radii and higher resolutions as the simulation proceeds.

The observation mentioned above is that unigrid finite difference codes are generally regarded as being significantly easier to develop than pseudospectral codes. Our interest, from a code-development standpoint, has been to provide an infrastructure whereby existing unigrid codes can be "endowed" with mesh refinement in a way which is somewhat automatic, such that the developer of the original unigrid code is spared the details of implementing mesh refinement. If the introduction of mesh refinement does not significantly alter the dynamics of the system, then one should be able to obtain results comparable to a high resolution unigrid run via an FMR run with appropriately placed fine grids which share the resolution of the unigrid run. In fact, this is the hope of this paper, and the criterion by which we evaluate our results obtained by FMR: ideally, the use of mesh refinement should produce results of comparable quality to a corresponding unigrid run, in terms of stability, accuracy and convergence behaviour. Thus our mesh refinement infrastructure could, in applicable cases, provide a service to the community of researchers who commonly develop unigrid finite difference codes, by providing these researchers a means by which to achieve more accurate results than their current computer allocations allow. It is in this spirit that we are making the FMR system, called Carpet (authored by Erik Schnetter, with refinements offered by several others), freely available as a driver thorn of the open-source Cactus computational infrastructure [31, 32, 33, 34, 35].

\section{OVERVIEW OF OUR FMR METHOD}

\section{A. Cactus and mesh refinement}

Cactus is an application framework that provides some computational infrastructure such as parallelisation and disk I/O, and lets users specify the modules, called "thorns", which contain all the "physics". The main difference between an application framework and a library of subroutines is control inversion, meaning that it is Cactus calling the users' routines, while the main program is part of Cactus. The part of Cactus that controls the creation of initial data and the time stepping is called the driver, which is a thorn that interacts with the Cactus scheduler in order to determine which routines are applied to which grid at what time. Control inversion has the important advantage that, by replacing the driver, one can change a Cactus application from unigrid to mesh refinement without rewriting any of the users' thorns.

In practice, the ability to use the same code with both unigrid and mesh refinement drivers places restrictions on the implementation of a routine. For most evolution thorns these modifications are at most minor. Some thorns, particularly those solving elliptic equations, require more substantial alterations. In most circumstances the restrictions imposed are only technical points, and it should be simple for any new code to be implemented to work with a unigrid or mesh refinement driver interchangeably. Our experience is also that analysis tools for e.g. wave extraction or apparent horizon finding continue to work almost without changes. 


\section{B. Mesh refinement method}

The mesh refinement driver that we use is called Carpet and is available together with the application framework Cactus. It uses the Berger-Oliger approach [1], where the computational domain as well as all refined subdomains consist of a set of rectangular grids. In particular, we base our scheme on the so-called "minimal Berger-Oliger" setup popularised by Choptuik [36]. In this simplified version of Berger-Oliger, the grid points are located on a grid with Cartesian topology, and the grid boundaries are aligned with the grid lines. In our version, we also allow fine grid boundaries to occur in between coarse grid points, as shown in Figure 1 (Note that this definition still allows, e.g., spherical coordinate systems.) Furthermore, there is a constant refinement ratio between refinement levels (described below).

We use the following notation. The grids are grouped into refinement levels (or simply "levels") $L^{k}$, each containing an arbitrary number of grids $G^{k}$. Each grid on refinement level $k$ has the grid spacing (in one dimension) $\Delta x^{k}$. The grid spacings are related by the relation $\Delta x^{k}=\Delta x^{k-1} / N_{\text {refine }}$ with the integer refinement factor $N_{\text {refine. An example is }}$ shown in Figure1 In what follows we will assume that $N_{\text {refine }}$ is always set to 2 . The base level $L^{0}$ covers the entire domain (typically with a single grid) using a coarse grid spacing. The base level need neither be rectangular nor connected. The refined grids have to be properly nested. That is, any grid $G^{k}{ }_{j}$ must be completely contained within the set of grids $L^{k-1}$ of the next coarser level, except possibly at the outer boundaries.

Although Cactus does allow both vertex and cell centred grids, current relativity thorns only use vertex centring. Hence Carpet currently only supports vertex centred refinement, i.e. coarse grid points coincide with fine grid points (where fine grid points exist).

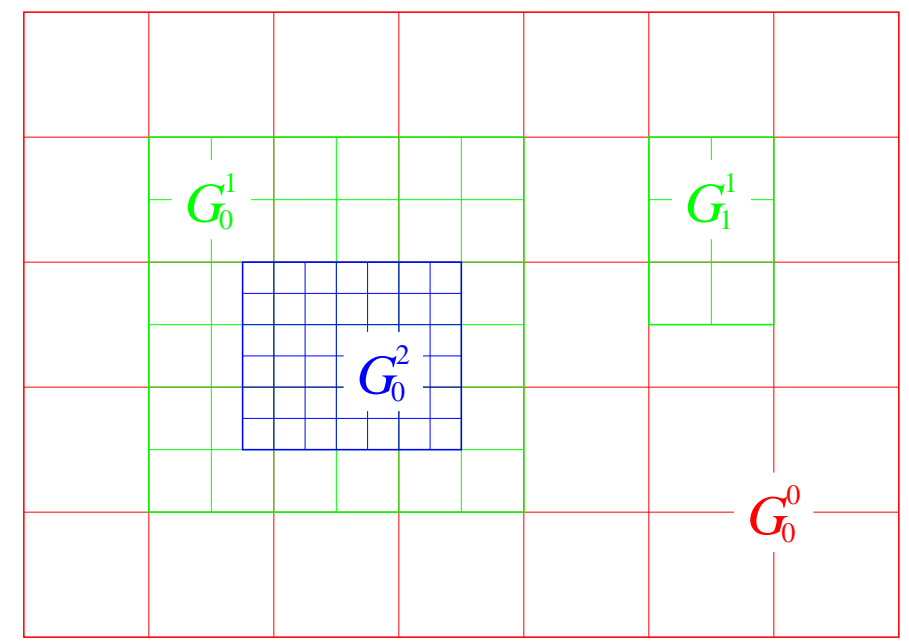

FIG. 1: Base level $G_{0}^{0}$ and two refined levels $G_{j}^{1}$ and $G_{j}^{2}$, showing the grid alignments and demonstrating proper nesting.

The times and places where refined grids are created and removed are decided by some refinement criterion. The simplest criterion, which is also indispensable for testing, is manually specifying the locations of the refined grids at fixed locations in space at all times. This is called fixed mesh refinement. A bit more involved is keeping the same refinement hierarchy, but moving the finer grids according to some knowledge about the simulated system, tracking some feature such as a black hole or a neutron star. This might be called "moving fixed mesh refinement". Clearly the most desirable strategy is an automatic criterion that estimates the truncation error, and places the refined grids only when and where necessary. This is what is commonly understood by adaptive mesh refinement. Carpet supports all of the above in principle, but we will only use fixed mesh refinement in the following. It should be noted that automatic grid placement is a non-trivial problem (see, e.g., [37, 38]).

\section{Time evolution scheme}

The time evolution scheme follows that of the Berger and Oliger [1] AMR scheme, in which one evolves coarse grid data forward in time before evolving any data on the finer grids. These evolved coarse grid data can then be used to provide (Dirichlet) boundary conditions for the evolution of data on the finer grids via prolongation, i.e. interpolation in time and space. This is illustrated in Figure 2 For hyperbolic systems, where a Courant-like criterion 
holds, a refinement by a factor of $N_{\text {refine }}$ requires time step sizes that are smaller by a factor $N_{\text {refine, }}$ and hence $N_{\text {refine }}$ time steps on level $k+1$ are necessary for each time step on level $k$. At time steps in which the coarse and fine grids are both defined, the fine grid data are restricted onto the coarse grid (via a simple copy operation) after it has been evolved forward in time. If there are more than two grid levels, then one proceeds recursively from coarsest to finest, evolving data on the coarsest grid first, interpolating this data in time and space along boundaries of finer grids, evolving the finer grid data, and restricting evolved data from finer to coarser grids whenever possible. This is illustrated in Figure 3

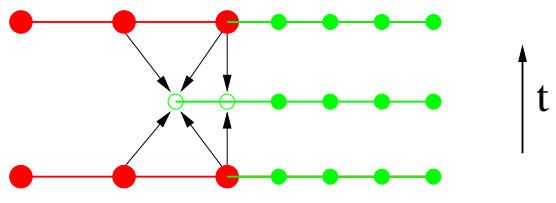

FIG. 2: Schematic for the prolongation scheme, in $1+1$ dimensions, for a two-grid hierarchy. The large filled (red) circles represent data on the coarse grid, and smaller filled (green) circles represent data on the fine grid. The arrows indicate interpolation of coarse grid data in space and time, necessary for the boundary conditions on the fine grid (explained in section $\amalg \mathrm{C}$.

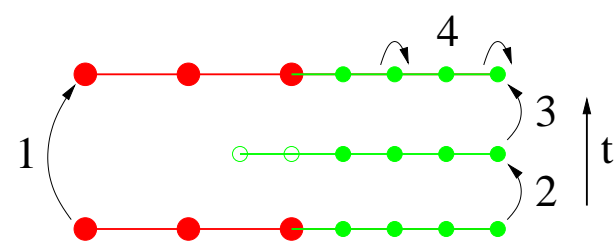

FIG. 3: Schematic for the time evolution scheme, in $1+1$ dimensions, for a two-grid hierarchy. The large filled (red) circles represent data on the coarse grid, and smaller filled (green) circles represent data on the fine grid. The algorithm uses the following order. 1: Coarse grid time step, 2 and 3: fine grid time steps, 4: restriction from fine grid to coarse grid. Since the fine grid is always nested inside a coarse grid, there are also coarse grid points (not shown) spanning the fine grid region (at times when the coarse grid is defined) at the locations of "every other" fine grid point; the data at these coarse grid points are restricted (copied directly) from the fine grid data.

For time evolution schemes that consist only of a single iteration (or step), the fine grid boundary condition needs to be applied only once. Most higher-order time integrations schemes, such as Runge-Kutta or iterative CrankNicholson, are actually multi-step schemes and correspondingly require the fine grid boundary condition to be applied multiple times. If this is not done in a consistent manner at each iteration, then the coarse and the fine grid time evolution will not couple correctly, and this can introduce a significant error. We explain this in more detail in Appendix $\mathrm{A}$

There are several ways to guarantee consistent boundary conditions on fine grids. Our method involves not providing any boundary condition to the individual integration substeps, but instead using a larger fine grid boundary, as demonstrated in Figure 4 That is, each of the integration substeps is formally applied to a progressively smaller domain, and the prolongation operation re-enlarges the domain back to its original size. Note that this "buffering" is done only for prolongation boundaries; outer boundaries are handled in the conventional way. Also, this is done only for the substeps due to the time integration scheme, so that the prolongation is applied at fine grid times when there is no corresponding coarse grid time. Note also that the use of buffer zones is potentially more computationally efficient.

We emphasise that the use of these buffer zones is not always necessary. To our knowledge the buffer zones are necessary only when the system of equations contains second spatial derivatives, and a multi-step numerical method is used for time integration. This issue arises for the BSSN system discussed below. We also give a simple example using the scalar wave equation in section IVA and appendix $\mathrm{A}$

\section{Inter-grid transport operators}

As described above, the interaction between the individual refinement levels happens via prolongation and restriction. For prolongation, Carpet currently supports polynomial interpolation, up to quadratic interpolation in time, which requires keeping at least two previous time levels of data. It also supports up to quintic interpolation in space, which requires using at least three ghost zones. We usually use cubic interpolation in space, which requires only two 


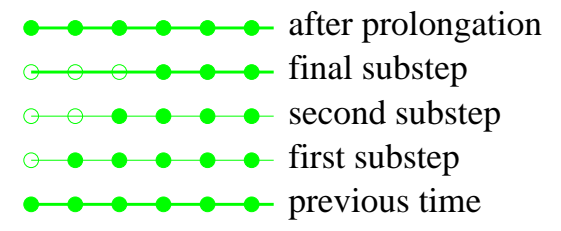

FIG. 4: Schematic for the "buffering" during time integration. Shown is the left edge of a refined region, which extends further to the right, which is integrated in time with a 3-step ICN method. At the filled points (in the interior), time integration proceeds as usual. The empty points (near the boundary) are left out, because no boundary condition is given during time integration. A prolongation after the time integration fills the empty points again. This whole scheme corresponds to either of the steps labelled 2 and 3 in Figure 3

ghost zones. (Quadratic interpolation in space introduces an anisotropy on a vertex centred grid.) For restricting, Carpet currently uses sampling (i.e., a simple copy operation). These transport operators are not conservative. Since our formulation of Einstein's equation (see below) is not in a conservative form, any use of conservative inter-grid operations offers no benefit. However, the transport operators can easily be changed. (For more discussion of the situations where conservative inter-grid operators are useful or not, see [39].)

\section{E. Initial data generation}

Initial data generation and time evolution are controlled by the driver Carpet. Initial data are created recursively, starting on the coarsest level $L^{0}$. This happens as follows: On refinement level $L^{k}$, the initial data routines are called. This fills the grids on this level. Then the refinement criterion is evaluated (which might be nothing more than a fixed mesh refinement specification). If necessary, grids on a finer level $L^{k+1}$ are created, and initial data are created there, and on all finer levels recursively. Then, the data from level $L^{k+1}$ are restricted to level $L^{k}$ to ensure consistency.

In many cases, the initial data specification is only valid for a single time $t=0$, such as when using a timesymmetric approach, or when solving an elliptic equation. However, for the time interpolation necessary during prolongation (see above), it may be necessary to have data on several time levels. One solution is to use only lower order interpolation during the first few time steps. We decided instead, according to the Cactus philosophy, that the data that are produced during the initial data creation should in principle be indistinguishable from data produced by a time evolution. Hence we offer the option to evolve coarse grid data backwards in time in order to provide sufficient time levels for higher order interpolation in time at fine grid boundaries. This ensures that no special case code is required for the first steps of the time evolution.

This initial data generation proceeds in two stages. First the data are evolved both forwards and backwards in time one step, leading to the "hourglass" structure illustrated Figure[5 This evolution proceeds recursively from coarsest to finest, so that all data necessary for time interpolation are present. Note that this would not be the case if we evolved two steps backwards in time, as there would not be enough data for the time interpolation for the restriction operation between these two steps.

In the end we must provide initial data only at times preceding the initial time $t=0$; i.e., the hourglass structure of Figure 5 is invalid as an initial data specification in Cactus. Therefore we perform in the second stage of this scheme one additional step backwards in time on each level, leading to initial data at the times $t_{0}, t_{0}-\Delta t^{k}$, and $t_{0}-2 \Delta t^{k}$ on each level $L^{k}$.

\section{PHYSICAL SYSTEM}

The set of equations we solve are described in detail in [40], and although we briefly review the material here, we suggest interested readers refer to the prior publication. The evolution system is that of Shibata-Nakamura [41] and Baumgarte-Shapiro [42], the so-called BSSN formulation. The physical quantities present in a typical ADM [43] evolution are the 3-metric $\gamma_{i j}$ and the extrinsic curvature $K_{i j}$. In the BSSN formulation, one instead evolves a different set of variables: $K_{i j}$ is decomposed into its trace $K$ and its trace-free part

$$
A_{i j} \equiv K_{i j}-\frac{1}{3} \gamma_{i j} K
$$




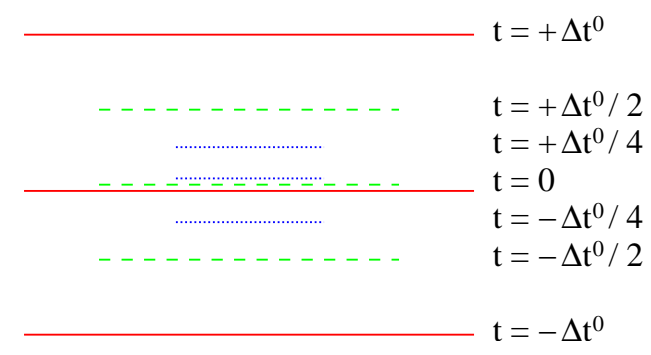

FIG. 5: Schematic for initial data scheme, in $1+1$ dimensions. Our use of quadratic interpolation in time requires three time levels of coarse grid data in order to provide boundary data for evolution on fine grids. To achieve this from the beginning of the evolution (without the use of a known continuum solution with which to "pre-load" these levels), we evolve our initial data (defined at $t=0$ ) both forwards and backwards one step in time. In this way, three time levels of coarse grid data are always available to provide boundary data along the edges of fine grids. The data at various times are denoted by fractions of the time step $\Delta t^{0}$ on the base grid. The coarsest grid is shown as by a solid (red) line, a finer grid by a long-dashed (green) line, and a still finer grid by a dotted (blue) line. (We perform some additional backwards evolution as well, which we describe in the main text. The essence of the scheme, however, is given here.)

and one applies a conformal transformation,

$$
\tilde{\gamma}_{i j}=e^{-4 \phi} \gamma_{i j}
$$

We choose $\phi$ such that the determinant of $\tilde{\gamma}_{i j}$, denoted by $\tilde{\gamma}$, is 1 .

Thus our evolved quantities are related to the ADM and physical quantities by

$$
\begin{aligned}
\phi & =\frac{1}{12} \log (\gamma) \\
\tilde{\gamma}_{i j} & =e^{-4 \phi} \gamma_{i j} \\
K & =\gamma^{i j} K_{i j} \\
\tilde{A}_{i j} & =e^{-4 \phi}\left(K_{i j}-\frac{1}{3} \gamma_{i j} K\right)
\end{aligned}
$$

One also creates a new evolved variable $\tilde{\Gamma}^{i}$, defined as

$$
\tilde{\Gamma}^{i}:=\tilde{\Gamma}_{j k}^{i} \tilde{\gamma}^{j k}
$$

The evolution equations for these variables are given by

$$
\begin{aligned}
\partial_{t} \phi= & -\frac{1}{6} \alpha K+\beta^{k} \partial_{k} \phi+\frac{1}{6} \partial_{k} \beta^{k} \\
\partial_{t} \tilde{\gamma}_{i j}= & -2 \alpha \tilde{A}_{i j}+\beta^{k} \partial_{k} \tilde{\gamma}_{i j}+\tilde{\gamma}_{i k} \partial_{j} \beta^{k}+\tilde{\gamma}_{j k} \partial_{i} \beta^{k}-\frac{2}{3} \tilde{\gamma}_{i j} \partial_{k} \beta^{k} \\
\partial_{t} K= & -D^{i} D_{i} \alpha+\alpha\left(\tilde{A}_{i j} \tilde{A}^{i j}+\frac{1}{3} K^{2}\right)+\beta^{i} \partial_{i} K \\
\partial_{t} \tilde{A}_{i j}= & e^{-4 \phi}\left[-D_{i} D_{j} \alpha+\alpha R_{i j}\right]^{T F}+\alpha\left(K \tilde{A}_{i j}-2 \tilde{A}_{i k} \tilde{A}_{j}^{k}\right) \\
& +\tilde{A}_{k j} \partial_{i} \beta^{k}+\tilde{A}_{k i} \partial_{j} \beta^{k}-\frac{2}{3} \tilde{A}_{i j} \partial_{k} \beta^{k} \\
\partial_{t} \Gamma^{i}= & -2 \partial_{j} \alpha \tilde{A}^{i j}+2 \alpha\left(\tilde{\Gamma}_{j k}^{i} \tilde{A}^{k j}-\frac{2}{3} \tilde{\gamma}^{i j} \partial_{j} K+6 \tilde{A}^{i j} \partial_{j} \phi\right) \\
& -\partial_{j}\left(\beta^{k} \partial_{k} \tilde{\gamma}^{i j}-\tilde{\gamma}^{k j} \partial_{k} \beta^{i}-\tilde{\gamma}^{k i} \partial_{k} \beta^{j}+\frac{2}{3} \tilde{\gamma}^{i j} \partial_{k} \beta^{k}\right),
\end{aligned}
$$

where $D_{i}$ is the covariant derivative corresponding to the 3-metric $\gamma_{i j}, R_{i j}$ is the three dimensional Ricci tensor, and the " $T F$ " superscript denotes the trace-free part of the enclosed expression.

The gauge conditions are given as follows. The lapse $\alpha$ is chosen as one of the Bona-Massó [44] family of slicings,

$$
\partial_{t} \alpha=-\alpha^{2} f(\alpha)\left(K-K_{0}\right)
$$


where $K_{0} \equiv K(t=0)$, and $f(\alpha)$ is chosen to give us either harmonic slicing $(f(\alpha)=1)$ or a " $1+\log$ " slicing $(f(\alpha)=2 / \alpha)$. In this paper the shift will be held constant at the analytic value in all cases.

We use the code described in [45, 46] and refer there for details of the finite differencing scheme used.

For certain problems a small amount of artificial dissipation is useful. We use a dissipation of the form

$$
\partial_{t} u=-\epsilon h^{4}\left(\partial_{x x x x}+\partial_{y y y y}+\partial_{z z z z}\right) u
$$

with the grid spacing $h$, which is described in [47]. Although this dissipation operator is typically employed only in systems with first order derivatives in time and space, we find that its use in the BSSN system (which has first order derivatives in time, and second order derivatives in space) is effective at reducing high-frequency oscillations (i.e., noise) in the simulations, but has little effect on the overall convergence behaviour.

\section{TESTS}

For simplicity we will present tests using only two levels containing one grid each, which we will refer to as the "coarse grid" and the "fine grid". The fine grid is a box contained in the larger coarse grid box, with the fine grid having a mesh spacing (in space and time) of half that of the coarse grid. The only limitation on the number of grids is the available computational resources, and we have successfully performed tests with up to 24 levels of refinement.

One of the principal criteria we use to evaluate the effectiveness of the FMR scheme is the requirement of secondorder convergence in the limit as the mesh spacing goes to zero. Thus we run a given simulation many times at different resolutions. In our examples we compare against the exact solution and check the convergence of the solution error. We show such tests only for the data on the coarsest grid, because the restriction operator ensures that the coarse grid and fine grid data are identical.

\section{A. Wave Equation: Periodic Boundaries}

We tested our code with a simple wave equation in flat space in Cartesian coordinates using several different kinds of initial data and boundary conditions. The first such test was that of sinusoidal plane waves in a 3D box with periodic boundary conditions. From a code-development standpoint, we simply took an existing set of subroutines for solving the wave equation in parallel and ran using the FMR driver instead of the usual unigrid driver. The formulation of the wave equation we used was a single equation with second order derivatives in both time and space, i.e.

$$
\partial_{t t} u=\partial^{i} \partial_{i} u,
$$

which we solved using a leapfrog-like scheme. Second order convergence was found, as shown in Figure 6

An alternative formulation of the wave equation uses second order derivatives in space but only first order in time. We write it in the form

$$
\begin{aligned}
& \partial_{t} u=v \\
& \partial_{t} v=\partial^{i} \partial_{i} u
\end{aligned}
$$

This formulation is comparable to the ADM (and BSSN) formulations of the Einstein equations. When this formulation of the scalar wave equation is evolved using ICN integration without buffer zones, the result is only first-order convergent, as shown using a one dimensional example in Figure 7 The same formulation evolved with buffer zones converges even at extremely high resolution as shown in Figure 8

Having demonstrated the need for careful handling of refinement boundaries, and having introduced buffer zones as an effective approach to that, we use buffer zones in all the remaining tests discussed in this paper.

\section{B. Wave Equation: Gaussian pulse}

We consider a Gaussian pulse that crosses a mesh refinement boundary, travelling from the fine into the coarse region. This is supposed to mimic the case of gravitational waves propagating from fine, inner grids and radiating out into coarser grids. 

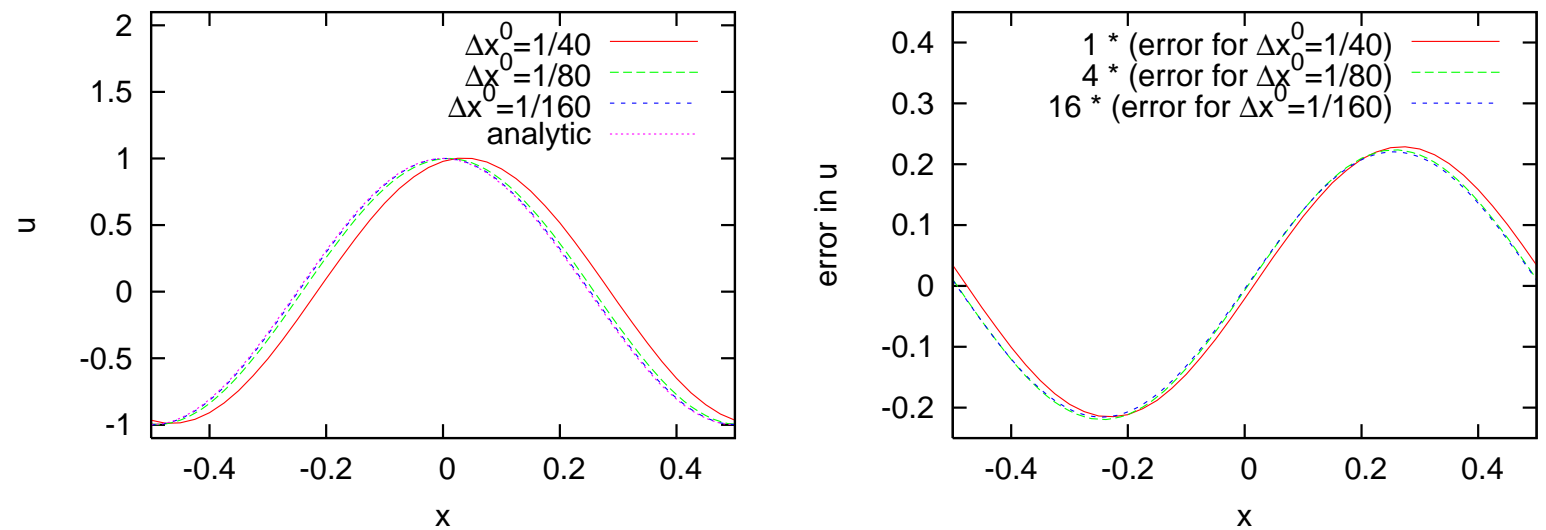

FIG. 6: Result at 50 crossing times in the evolution of the wave equation with periodic boundaries, for two-level runs at three different resolutions $(1 / 40,1 / 80$, and $1 / 160)$. Here we show only data for the coarse grid; the finer grid covers the box $x_{i} \in$ $[-0.25,+0.25]$. The left panel shows slices along the $x$-axis of the (3D) function $u$ found numerically, as well as the analytic (i.e. continuum) solution. (In this graph the highest resolution data and the analytic solution appear to lie on top of each other.) One can see a resolution-dependent phase shift in $u$. The right panel shows the solution error, defined as the difference between the $u$ obtained numerically and the continuum solution. These error graphs have been scaled to demonstrate the second order convergence of the numerical results.
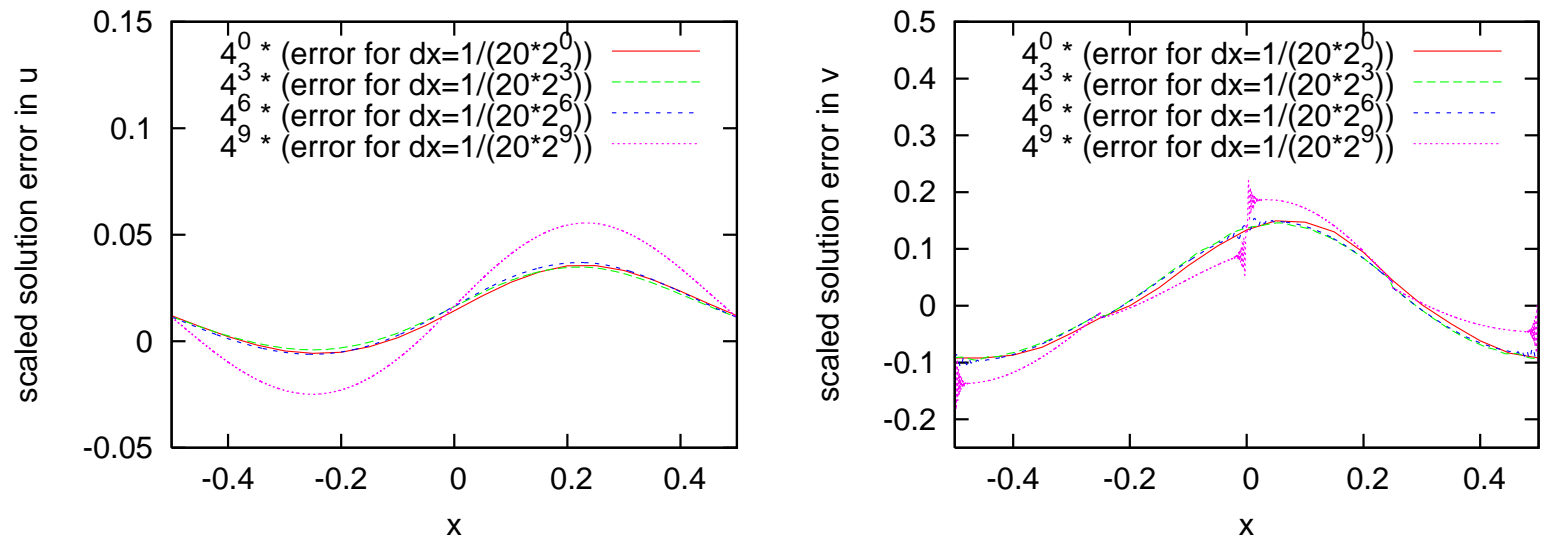

FIG. 7: Scaled solution errors for the 1-D scalar wave equation at 0.75 crossing times, discretised without buffer zones. The left panel shows the error in the solution $u$, the right panel in its time derivative $v$. At low resolutions, i.e. from $1 / 20$ up to about $1 / 1280$, the scheme seems to be second-order convergent. However, at higher resolutions it becomes clear that this is not the case. At lower resolutions, the refinement boundaries are visible as small discontinuities in the error of $v$ at $x= \pm 0.25$. At higher resolutions, the discontinuity develops an oscillating tail and propagates through the simulation domain. It is instructive to see that 8 convergence test levels were necessary to see this behaviour numerically. Compare this against Figure 8

We use an effectively one dimensional domain (planar symmetry in 3D) with $x \in[-0.5,+0.5]$, and a coarse grid resolution of $\Delta x^{0}=1 / 100$. The region $x>0$ is refined by a factor of 2 . The Gaussian pulse starts in the refined region and travels to the left. Figure 9 shows the pulse after it has crossed the interface, and compares the result to two unigrid simulations. The errors that are introduced at the refinement boundary are very small and converge to second order. In particular, at this resolution about $10^{-3}$ of the original pulse is reflected by the refinement boundary.

\section{Wave Equation: $1 / r$, with excision}

Another test we performed was the solution of the wave equation (15) using initial data which scaled inversely with $r$, the distance from the origin. This was conceived as a useful step before moving on to black holes [48], as the "puncture data" [49] we use for the black holes has elements which scale as $1 / r$ in the vicinity of the puncture. Other systems with isolated central masses may also be expected to have elements which scale in a similar fashion. The $1 / r$ 

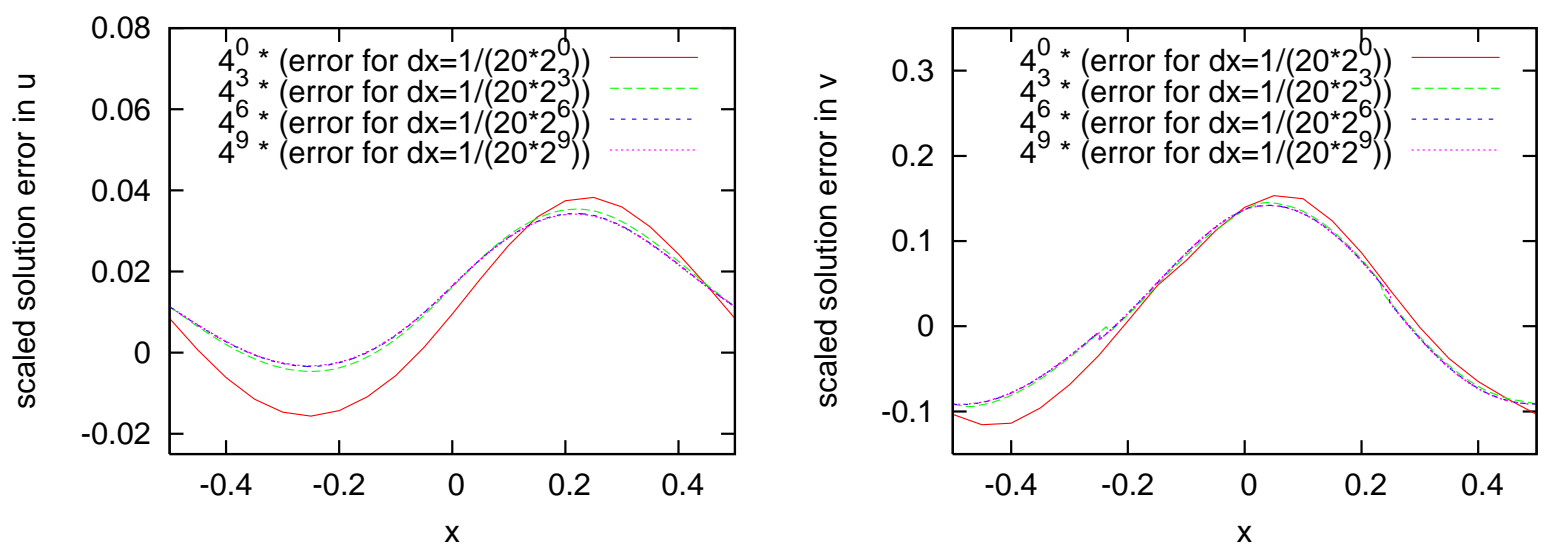

FIG. 8: Scaled solution errors for the 1-D scalar wave equation at 0.75 crossing times, discretised with buffer zones. The left panel shows the error in the solution $u$, the right panel in its time derivative $v$. The refinement boundaries are visible as small discontinuities in the error of $v$ at $x= \pm 0.25$. The results are second-order convergent up to extremely high resolutions. It can also be seen that the resolution $1 / 20$ is clearly not yet in the convergence regime.
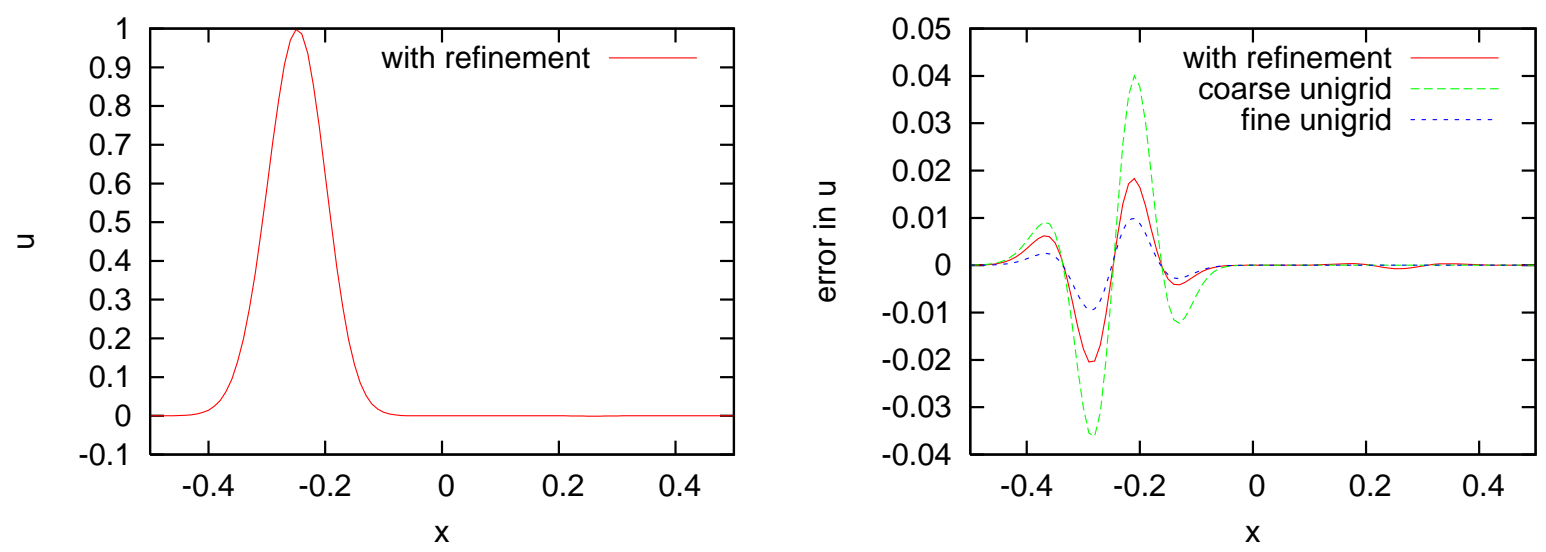

FIG. 9: The left panel shows a Gaussian pulse that has travelled through a refinement boundary located at $x=0$. The region $x>0$ is refined. The right panel shows the difference to the analytic solution, and shows two unigrid runs for comparison. The resolutions of the unigrid runs correspond to the coarse and the fine regions in the mesh refinement run. As expected, the error in the run with refinement is in between the errors of the two unigrid runs. The reflected part of the pulse is very small; it has an amplitude of only $10^{-3}$ and is thus much smaller than the discretisation error in the pulse itself.

data are a static solution of the wave equation in 3D, and are compatible with the standard Sommerfeld outgoing boundary condition. To handle the singularity at the centre, we "excise" the centre of the computational domain by choosing some inner boundary at a finite excision radius from the centre and filling the interior region with prescribed data. Thus in this test we also have a test of the use of FMR in the presence of excision, which is commonly used for the interiors of black holes in analogous simulations. We perform no evolution within this excised region.

We use a full 3D grid with $x_{i} \in[-1,+1]$ and coarse grid resolutions of $\Delta x^{0}=1 / 32$ and $\Delta x^{0}=1 / 64$. The region $x_{i} \in[-0.5,+0.5]$ is refined by a factor 2 , and the region $\left|x_{i}\right| \leq 0.125$ is excised. Graphs of the error at two different times in the evolution are shown in Figure 10, which also shows corresponding unigrid runs for comparison. We see that the solution is fully convergent, and similar to the corresponding unigrid results in the region of refinement. It is interesting to note that even outside this region, the FMR and unigrid results are very similar for the "transient" shown in the left frame, however the late time ("stationary") behaviour shown in the right frame reveals a notable difference between the FMR and unigrid results outside the refined grid.

Having demonstrated the existence of convergent solutions of the wave equation for oblique angles of incidence to refinement boundaries (in the $1 / r$ case), and convergent solutions in which the amplitude of the reflected wave is significantly (roughly three orders of magnitude) less than the amplitude of the transmitted wave, we now move on to full solutions of Einstein's equations. For more detailed calculations of reflection and transmission effects at 

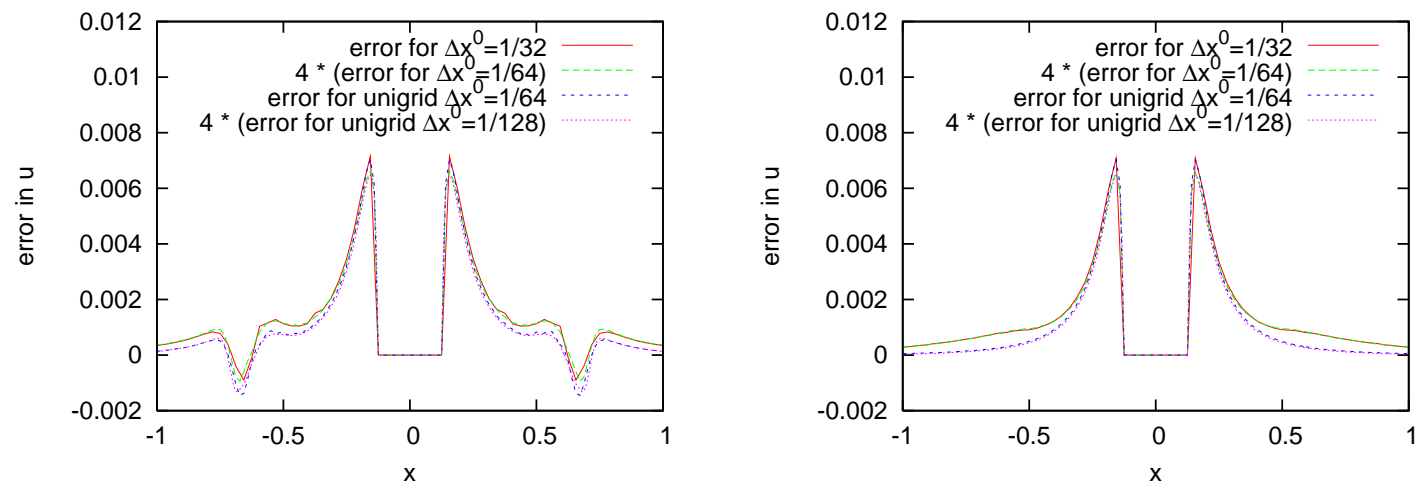

FIG. 10: Solution error for evolutions of $1 / r$ data at times $t=0.625$ (left frame) and $t=5.0$ (right frame), using radiation (Sommerfeld) boundary conditions. Here we show only the base grid; the refined grid covers the domain $x_{i} \in[-0.5,+0.5]$. The region $\left|x_{i}\right| \leq 0.125$ is excised. We see in the left frame that an initial transient moving outward from the refined region is still resolved in a second-order-convergent manner after it passes through the refinement boundary. At later times, the error settles down to the profile shown in the right frame. As expected, the FMR results compare favourably with the unigrid results in the region covered by the refined grid. (Note that, outside the refined region, the errors shown for the FMR results have effectively been multiplied by a factor of 4 relative to the comparable unigrid results, giving the impression of greater disagreement than is really present.)

refinement boundaries see [12, 13].

\section{Robust Stability}

We have applied a robust stability test [50, 51, 52] to the BSSN formulation. This is a test that is meant to supplement and extend an analytic stability analysis, especially in cases where such an analysis is difficult or impossible (because the equations contain too many terms). A numerical test has the advantage that it tests the complete combination of evolution equations, gauge conditions, boundary conditions, as well as the discretisation and the implementation, and (in our case) the mesh refinement scheme. Thus, while a numerical test is not as reliable as an analytically obtained statement, it is able to cover more general cases.

The robust stability test proceeds in three stages of increasing difficulty:

Stage I: Minkowski (or some other simple) initial data with small random perturbations. The simulation domain is a three dimensional box with periodic boundary conditions. The perturbations should be linear, so we chose a maximum amplitude of $10^{-10}$. The periodicity means that there is effectively no boundary, so that this stage is independent of the boundary condition. A code is deemed to be robustly stable if it shows at most polynomial growth and if the growth rate is independent of the grid resolution. This is different from other definitions of stability, where exponential growth is often deemed to be stable if the rate of exponential growth is independent of the grid resolution.

Stage II: The same as stage I, except that the boundaries in the $x$ direction are now Dirichlet boundaries. In addition to the noise in the initial data, noise is also applied to these Dirichlet boundaries. This tests the consistency of the formulation with the boundary conditions, but without the complications of edges and corners.

Stage III: The same as stage II, except that there are now Dirichlet boundary conditions in all directions. This tests whether edges and corners are handled correctly by the combination of the formulation and boundary conditions.

In accordance with [52] we chose an effectively one dimensional, planar symmetric domain that extends almost only in the $x$-direction with $x \in[-0.5,+0.5]$. The domain has only 3 grid points in the $y$ and $z$ directions. Thus we have to omit stage III of the test here. We used a resolution of $1 / 50$, and refined the centre of the domain. Although the domain was thus essentially one dimensional, the simulation was performed with the full three dimensional code.

Figure 11 shows the $L_{\infty}$-norm of the Hamiltonian constraint versus time for 1000 crossing times for the stages I and II for three different resolutions. All test were run without artificial dissipation. The results show that our implementation is robustly stable. 

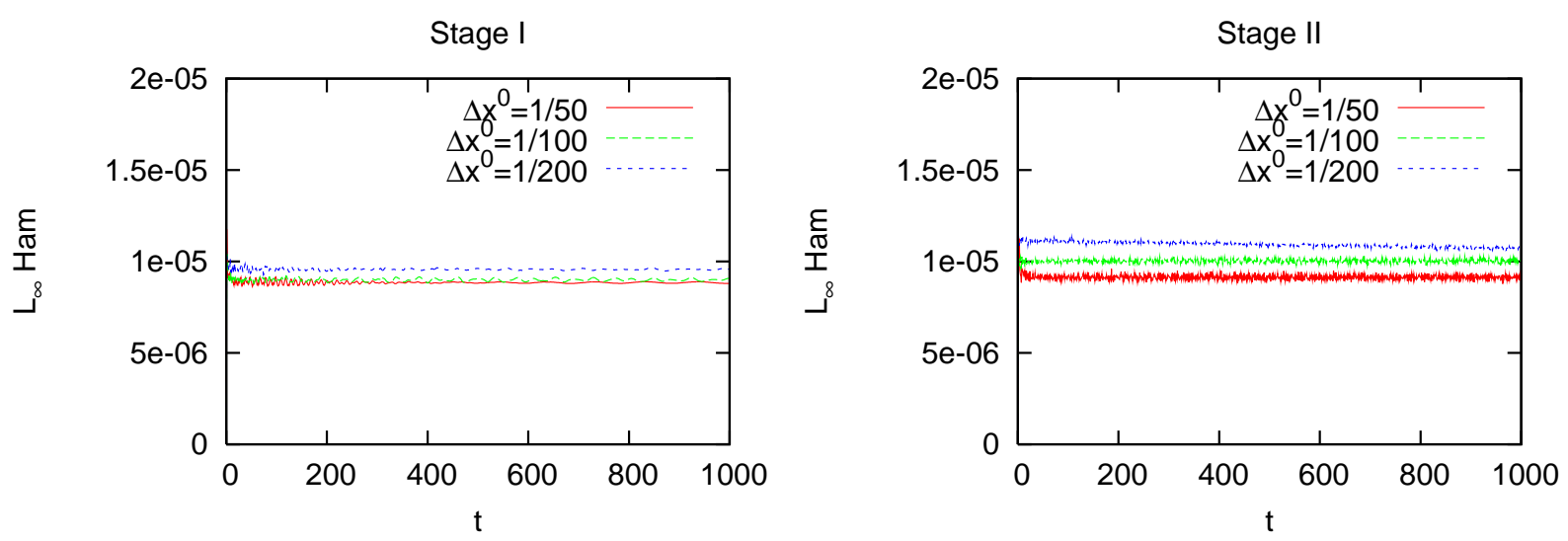

FIG. 11: $L_{\infty}$-norm of the Hamiltonian constraint vs. time for the robust stability test. The left hand side graph shows stage I where all boundaries are periodic. The right hand side graph shows stage II, which has noisy Dirichlet boundaries in the $x$ direction. The domain is essentially one dimensional with $x \in[-0.5,+0.5]$, and periodic in the $y$ and $z$ directions. The refined grid covers the region $x \in[-0.25,+0.25]$. The constraint violation in corresponding unigrid runs (not shown) has the same magnitude.

\section{E. Gauge Wave}

As another test of the BSSN system we implemented the gauge wave of [52]. From the typical Minkowski coordinates $\{\hat{t}, \hat{x}, \hat{y}, \hat{z}\}$, one defines new coordinates $\{t, x, y, z\}$ via the coordinate transformation

$$
\begin{aligned}
& \hat{t}=t-\frac{A d}{4 \pi} \cos \left(\frac{2 \pi(x-t)}{d}\right) \\
& \hat{x}=x+\frac{A d}{4 \pi} \cos \left(\frac{2 \pi(x-t)}{d}\right) \\
& \hat{y}=y \\
& \hat{z}=z,
\end{aligned}
$$

where $d$ is the size of the simulation domain. In these new variables, the 4-metric is

$$
d s^{2}=-H d t^{2}+H d x^{2}+d y^{2} d z^{2},
$$

where

$$
H=H(x-t)=1-A \sin \left(\frac{2 \pi(x-t)}{d}\right) .
$$

This test provides us with an exact solution to which we can compare our numerical results. In addition to the exact values $\alpha=\sqrt{H}$ and $g_{x x}=H$, we will compare the extrinsic curvature $K_{i j}$, for which the only nonzero component is

$$
K_{x x}=-\frac{\pi A}{d \sqrt{H}} \cos \left(\frac{2 \pi(x-t)}{d}\right) .
$$

Since $\beta^{i}=0$ in the analytic solution we do not evolve the shift but keep it set to zero at all times.

For this simulation we find it useful to add dissipation to the evolution equations to suppress high frequency noise at very high resolutions. The reason is evident from unigrid simulations at high resolutions, as demonstrated in Figure 12

The simulation domain was set up in the same way as in [52], which is also the same as is used in Section IVD That is, the simulation domain extended almost only in the $x$ direction with $x \in[-0.5,+0.5]$. The domain has only 3 grid points in the $y$ and $z$ directions. All boundaries are periodic. Although the domain is thus essentially one dimensional, the simulation was performed with the full three dimensional code. Figure 13 shows the results after 5 crossing times for the metric component $g_{x x}$, comparing refinement and unigrid runs. We see perfect second order convergence. 


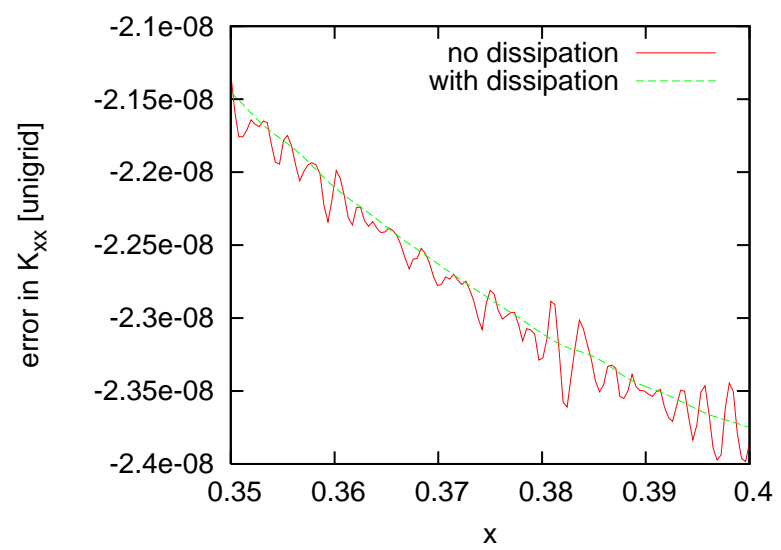

FIG. 12: Error in the extrinsic curvature component $K_{x x}$ along a small part of the $x$ axis for a unigrid simulation after 0.25 crossing times. The resolution, $\Delta x=1 / 2560$, is rather high. The noise (that was not present in the initial data) can be significantly reduced by dissipation. It is surprising to see that a unigrid simulation with the plain BSSN formulation shows this behaviour; this might point to an instability in the system of equations.
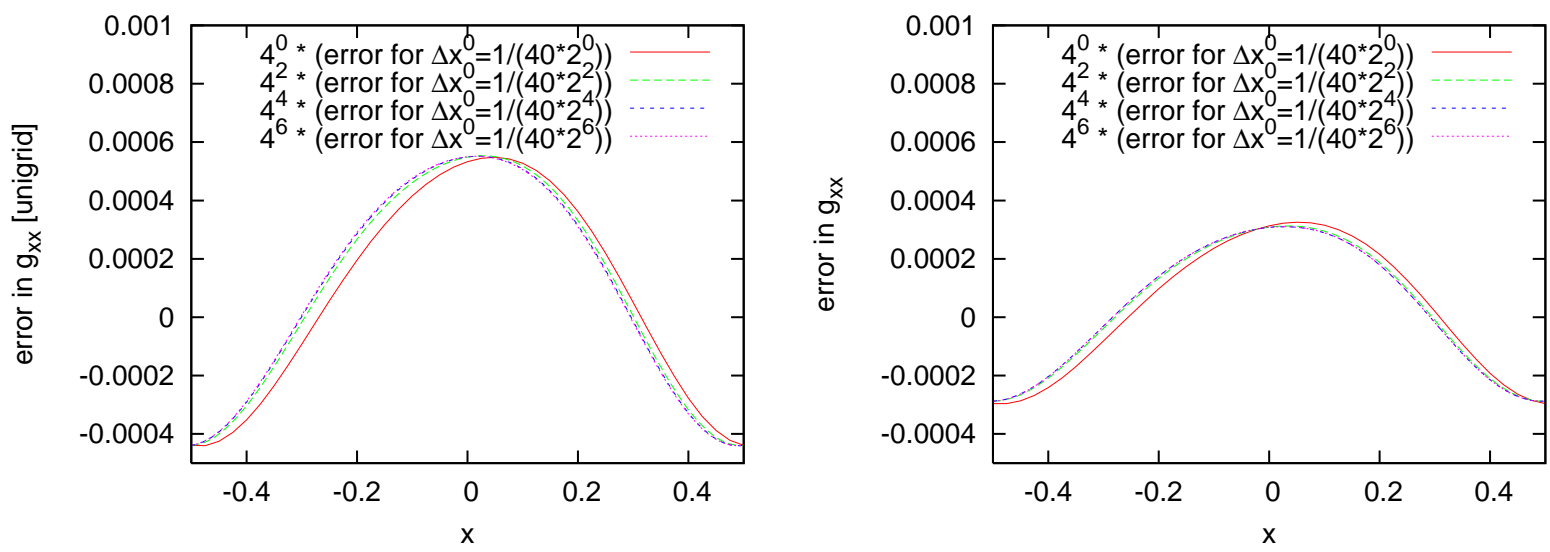

FIG. 13: Errors for a uniform grid (left) and with mesh refinement (right) after 5 crossing times for the three-metric component $g_{x x}$ in the gauge wave test case. The errors have been scaled with the resolution according to second order convergence.

\section{F. Schwarzschild Black Hole with Excision}

Next, we evolved a static Schwarzschild black hole in Kerr-Schild coordinates which are manifestly static. The line element is

$$
d s^{2}=-\left(1-\frac{2 M}{r}\right) d t^{2}+\left(\frac{4 M}{r}\right) d t d r+\left(1+\frac{2 M}{r}\right) d r^{2}+r^{2} d \Omega^{2}
$$

where $M$ is the mass of the black hole. We choose to use excision to handle the singularity. We use the excision method together with one of the tests described in [45]. The shift is held static at the analytic solution and the lapse is evolved using $1+\log$ slicing. For simplicity we do not search for an apparent horizon but merely excise those points within a cube with corners at $1 M$. This small excision region removes the divergences due to the singularity while retaining some of the steep gradients. However, as noted by [53, 54], this does not guarantee that the future light cone at the excision boundary is contained within the excised region.

We evolve only one octant of the grid to take advantage of the symmetries present. We use a coarse grid spacing of $\Delta x^{0}=0.4 M$ and $\Delta t^{0}=0.1 M$ with $29^{3}$ points, giving an outer boundary at $10 M$ (ignoring two symmetry points and two outer boundary points). The fine grid also contains $29^{3}$ points. The points are not staggered about the origin, so there is always a grid point at $r=0$ which must be excised.

We compare the runs using mesh refinement with simulations using the unigrid code as described in [45, 46]. The coarse unigrid test is identical to the coarse grid in the simulation using refinement. That is, $29^{3}$ points are used 
with a grid spacing of $\Delta x=0.4 M$ giving an outer boundary at $10 M$. For the medium unigrid run the resolution is doubled whilst the outer boundary location is held fixed, giving the same effective resolution near the excision region as the simulation using refinement. That is, $55^{3}$ points are used with a grid spacing of $\Delta x=0.2 M$. To compare with the high resolution run using refinement we also perform a unigrid run with the same effective resolution. That is, $105^{3}$ points are used with a grid spacing of $\Delta x=0.1 \mathrm{M}$.

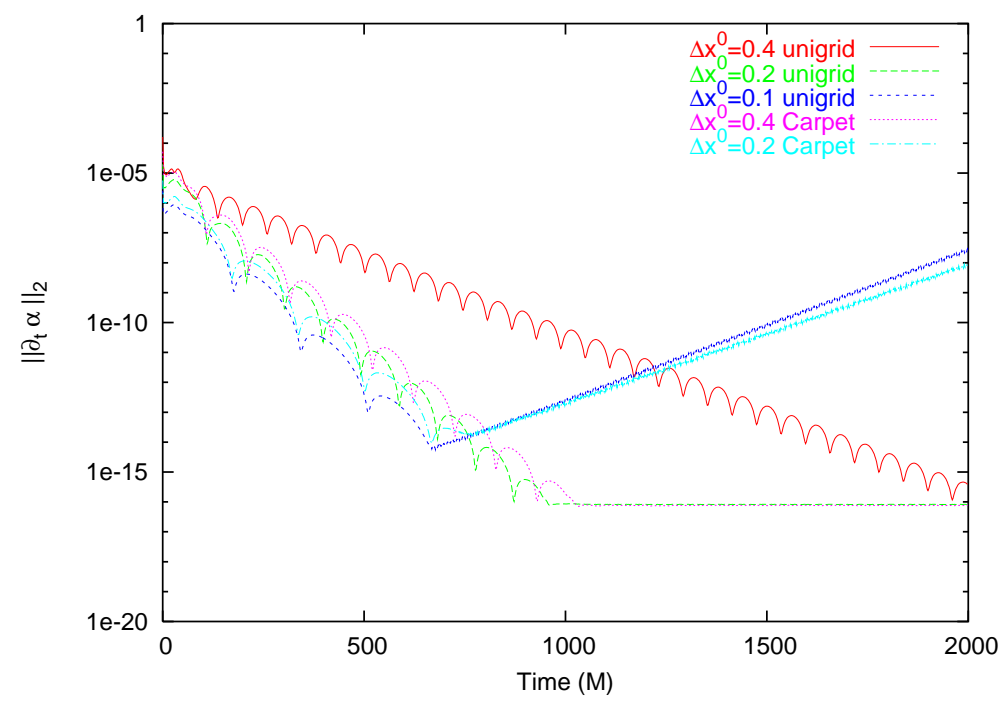

FIG. 14: The r.m.s. of the change in the lapse with time. As in [45] we see an exponentially damped oscillation as the system settles down. However, at sufficiently high resolution an instability sets in. This appears to come from floating point round-off error at the initial time and is clearly not caused by mesh refinement.

Results showing the norm of the change of the lapse in time are shown in Figure14 Firstly we note that the use of refinement combined with an excision boundary has no qualitative effect on the simulation. As in [45] the change in the lapse shows an exponentially damped oscillation in the absence of instabilities.

However, in this case we do see an exponentially growing instability which sets in only at the highest resolutions. By tracing back the magnitude of this mode we see that it appears to come from floating point round-off error at the initial time. It appears in runs both with and without mesh refinement and the growth rate is the same in both cases. The origin of this instability is unclear, especially as very similar simulations with the same resolutions were shown to be stable in [45]. However, the important point for this paper is that the stability of the simulations are independent of the mesh refinement.

The convergence of the Hamiltonian constraint at $t=500 \mathrm{M}$ is shown in figure 15, which should be compared with Figure 3 in [45]. Second order convergence for the unigrid and mesh refinement simulations are clear away from the excision boundary, whilst at the excision boundary the convergence is not so obvious due to the low resolution. The error in the mesh refinement runs is comparable to the unigrid runs with the same effective resolution, although only near the excision boundary are the full benefits seen. Given the computational resources required, illustrated in Table【 the benefits of mesh refinement are clear.

\begin{tabular}{|c|c|c|c|c|}
\hline $\begin{array}{c}\text { Effective } \\
\text { resolution (M) }\end{array}$ & Grid size & $\begin{array}{c}\text { Refinement } \\
\text { levels }\end{array}$ & $\begin{array}{c}\text { Memory } \\
\text { use (MB) }\end{array}$ & $\begin{array}{c}\text { Real (user) time } \\
(\mathrm{s})\end{array}$ \\
\hline 0.4 & 25 & 1 & 58 & $18.0(17.6)$ \\
0.2 & 25 & 2 & 114 & $85.5(84.7)$ \\
0.2 & 50 & 1 & 303 & $222.1(219.4)$ \\
0.1 & 50 & 2 & 695 & $957.6(950.8)$ \\
0.1 & 100 & 1 & 2024 & $3337.9(3309.9)$ \\
\hline
\end{tabular}

TABLE I: Computational resources required to evolve the single black hole with excision to $t=2 M$ with various grid sizes. The results using refinement give comparable results to unigrid results with the same effective resolution whilst using approximately $30 \%$ of the resources. These results used a $2.8 \mathrm{GHz}$ Intel Xeon processor with the Intel version 7 compilers. 


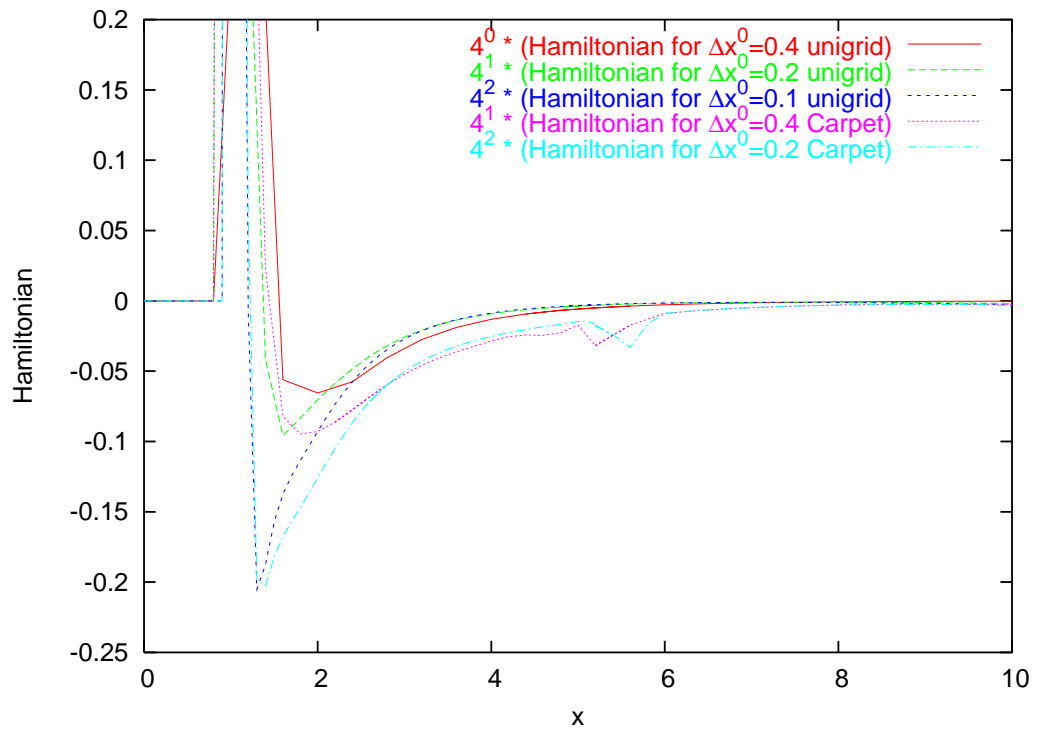

FIG. 15: Second order convergence of the Hamiltonian constraint for the Schwarzschild black hole at $t=500 M$. For the results using mesh refinement we plot the results using the finest possible level, causing a "jump" at the interface between the fine and coarse grid. We see that the mesh refinement simulations converge to second order except at the excision boundary. The simulation with refinement has comparable accuracy as the unigrid run with the same effective resolution near the excision boundary, but is less accurate away from this region.

\section{CONCLUSIONS}

We have shown comparisons of results obtained via fixed mesh refinement (FMR) techniques and via standard unigrid methods. Ideally, the appropriate use of FMR should produce results very similar in accuracy to the corresponding unigrid results, while retaining the stability and convergence properties of the unigrid simulations. "Corresponding" in this case implies that the unigrid simulation has the same resolution as the finest grid of the FMR simulation. This ideal is only expected to hold for simulations where most of the "interesting behaviour" occurs in a limited region which is covered by refined grids, such as in simulations of compact objects.

We find that particular care must be taken at boundaries between coarse and fine grids, in order to retain stability and convergence. In cases where the system contains second spatial derivatives and a multistep time integration method is used, we introduce buffer zones to guarantee the convergence of the mesh refinement implementation. The need for these buffer zones can be clearly seen in very fine resolution simulations of the wave equation when written in "mixed" form (with second spatial derivatives and first derivatives in time). The same effects are seen in simulations of gauge waves even at intermediate resolutions when using the BSSN formulation in a similar mixed form.

In order to use parabolic interpolation in time, even from the initial time, we designed a scheme by which data is initially evolved forwards and backwards in time. This means that the fine grid evolution can begin with three time levels of coarse grid data with which to do the time interpolation.

We are able to obtain results from FMR simulations which compare favourably with corresponding unigrid simulations for the following systems: the wave equation with $1 / r$ data and excision near the origin, robust stability tests, a nonlinear gauge wave, and a Schwarzschild black hole with excision. The robust stability tests indicate that numerical noise combined with inter-grid transport operations will not lead to exponential blow ups, and that the introduction of refinement boundaries does not produce instabilities. Both the wave equation test and the gauge wave test show that proper second order convergence can be obtained irrespective of waves propagating through the interfaces between coarse and fine grids. For the case of the Schwarzschild black hole, although we do see an instability at high resolutions, this instability is present in the unigrid system as well. At lower resolutions, we see stable behaviour showing close agreement between FMR and unigrid results.

There are few other implementations of mesh refinement in more than one dimension in numerical relativity with which to compare. The method of [18] is very close to ours. However, it is an axisymmetric code designed to evolve relativistic scalar fields. The code presented in [25] uses the ADM formalism (which is known to be unstable) and exhibits problems at the refinement boundaries. In the implementation of [13] which uses Paramesh ([55]), all refinement levels are evolved using the same timestep size. This avoids the difficulties that we observe in ob- 
taining convergence at refinement boundaries. However it may be less efficient, especially where a large range of refinement levels is employed. Our implementation provides both the full efficiency of the Berger-Oliger method and is a generic interface allowing the simple implementation of other formulations and systems such as relativistic hydrodynamics.

Finally, we note that the evolutions described in this paper were performed by taking routines for unigrid simulations of each physical system of interest, and after only slight modifications to these routines, the original unigrid driver was exchanged for the "Carpet" FMR driver. The application of FMR techniques to existing unigrid systems is thus something which, from a code development viewpoint, can be performed generally. A clear advantage of this approach is that most existing analysis tools (such as for wave extraction or apparent horizon finding) will continue to work. Thus we invite other researchers to make use of our freely available code to perform their finite-differencebased simulations in an FMR setting, and thus achieve higher accuracies with less computing resources than their current unigrid simulations may require.

\section{Acknowledgments}

We thank Bernd Brügmann and Nils Dorband for their early contributions to the work on the wave equation in FMR, Tom Goodale, Gabrielle Allen and the Cactus team for their assistance regarding the infrastructure development of Cactus which makes FMR possible, and Edward Seidel for much encouragement over the past years. We also thank our collaborators in Wai-Mo Suen's WuGrav group, with which we shared an early version of the code. Finally we thank Richard Matzner, Bernd Brügmann, and Denis Pollney for their helpful comments regarding the preparation of this manuscript. Work on this project was funded by the AEI, the DFG's Collaborative Research Centre SFB 382, and by NSF grant PHY 0102204.

\section{APPENDIX A: COUPLING COARSE AND FINE GRID TIME EVOLUTION}

When certain formulations of the equations are evolved with multi-step time integration methods, we find that the standard Berger-Oliger approach leads to a loss of convergence at the boundaries of fine grids. We will consider only second order methods here. We will argue that the use of Berger-Oliger style prolongation to find boundary conditions for refined grids at the intermediate steps causes a second order truncation error after a fixed number of time steps. Therefore the global error will be first order for a hyperbolic system where to integrate to a fixed time the number of timesteps increases with the grid resolution.

We first give an example, considering a simple one dimensional example on an infinite domain. The wave equation

$$
\partial_{t}^{2} u=\partial_{x}^{2} u
$$

can be reformulated into a system that is first order in time, but still second order in space:

$$
\begin{aligned}
& \partial_{t} u=v \\
& \partial_{t} v=\partial_{x}^{2} u .
\end{aligned}
$$

Discretising this system on a uniform grid in space and time, we represent the function $u$ and $v$ through

$$
\begin{aligned}
& u(t, x)=U_{i}^{n} \\
& v(t, x)=V_{i}^{n}
\end{aligned}
$$

at the collocation points $t=n \Delta t, x=i \Delta x$, where $n$ and $i$ number the grid points in the temporal and spatial direction, and $\Delta t$ and $\Delta x$ are the temporal and spatial grid spacings, respectively. The ratio $\alpha=\Delta t / \Delta x$ is the CFL factor. $h$ will be used to refer to terms that are order $\Delta x$ or $\Delta t$.

For the spatial discretisation of the operator $\partial_{x}^{2}$ we will consider the second order centred differencing stencil

$$
D_{x}^{2} F(x):=\frac{1}{(\Delta x)^{2}}(F(x-\Delta x)-2 F(x)+F(x+\Delta x)) .
$$

For time integration we consider one form of the ICN (iterative Crank-Nicholson) method. It consists first of an Euler step

$$
T_{E}\left[F_{0}\right]:=F_{0}+\Delta t \partial_{t} F_{0}
$$


and then several (in our case, two) ICN iterations

$$
T_{I}\left[F_{0}, F\right]:=F_{0}+\Delta t \partial_{t} \frac{1}{2}\left(F_{0}+F\right)
$$

so that the overall ICN integration with 2 iterations is given by

$$
T_{\mathrm{ICN}(2)}\left[F_{0}\right]:=T_{I}\left[F_{0}, T_{I}\left[F_{0}, T_{E}\left[F_{0}\right]\right]\right] .
$$

Note that there exist several slightly different variants of the ICN method.

This discretisation is correct up to second order, which means that for a fixed CFL factor $\alpha$, the discretisation error is $O\left(h^{2}\right)$. This means also that functions $f(t, x)$, which depend on $t$ and $x$ in a polynomial manner with an order that is not higher than quadratic, are represented exactly, i.e. without discretisation error. (Such functions $f$ can be written as

$$
f(t, x):=\sum_{p=0}^{2} \sum_{q=0}^{2} C_{p q} t^{p} x^{q}
$$

with constant coefficients $C_{p q}$.)

\section{Performing a single step}

It is illustrating to perform a time integration step by step. We will use for that the solution of the wave equation

$$
\begin{aligned}
& u(t, x)=\frac{1}{2} t^{2}+\frac{1}{2} x^{2} \\
& v(t, x)=t
\end{aligned}
$$

which will test whether the formulation is indeed capable of representing all functions of the form (A10).

Starting from the exact solution at time $t_{0}$,

$$
\begin{aligned}
& U\left(t_{0}, x\right)=\frac{1}{2} t_{0}^{2}+\frac{1}{2} x^{2} \\
& V\left(t_{0}, x\right)=t_{0}
\end{aligned}
$$

the result of the Euler step of the ICN integration is

$$
\begin{aligned}
U^{(0)}\left(t_{0}+\Delta t, x\right) & =U\left(t_{0}, x\right)+\Delta t V\left(t_{0}, x\right) \\
& =\frac{1}{2} t_{0}^{2}+\frac{1}{2} x^{2}+(\Delta t) t_{0} \\
V^{(0)}\left(t_{0}+\Delta t, x\right) & =V\left(t_{0}, x\right)+\Delta t D_{x}^{2} U\left(t_{0}, x\right) \\
& =t_{0}+\Delta t
\end{aligned}
$$

where $F^{(k)}$ denotes the result after $k$ ICN iterations. In these expressions, $U^{(0)}\left(t_{0}+\Delta t, x\right)$ differs from the true solution $U\left(t_{0}+h, x\right)$ by a term $O\left(h^{2}\right)$.

The first ICN iteration then leads to

$$
\begin{aligned}
U^{(1)}\left(t_{0}+\Delta t, x\right) & =U\left(t_{0}, x\right)+\Delta t \frac{1}{2}\left(V\left(t_{0}, x\right)+V^{(0)}\left(t_{0}+\Delta t, x\right)\right) \\
& =\frac{1}{2} t_{0}^{2}+\frac{1}{2} x^{2}+\Delta t t_{0}+\frac{1}{2}(\Delta t)^{2} \\
& =\frac{1}{2}\left(t_{0}+\Delta t\right)^{2}+\frac{1}{2} x^{2} \\
V^{(1)}\left(t_{0}+\Delta t, x\right) & =t_{0}+\Delta t \frac{1}{2}\left(D_{x}^{2} U\left(t_{0}, x\right)+D_{x}^{2} U^{(0)}\left(t_{0}+\Delta t, x\right)\right) \\
& =t_{0}+\Delta t
\end{aligned}
$$

which is already the correct result. The second ICN iteration does not change the above expressions. 


\section{Mesh refinement}

Let us now introduce mesh refinement, so that the spatial and temporal resolution is not uniform any more. Let the grid points be staggered about the origin, so that the grid points with $x<0$ have a coarse spatial grid spacing $2 \Delta x$, and the grid points with $x>0$ have a fine spatial grid spacing $\Delta x$. Let the CFL factor $\alpha$ remain uniform, so that the temporal grid spacing is correspondingly $2 \Delta t$ for $x<0$ and $\Delta t$ for $x>0$. We will use the Berger-Oliger time stepping scheme, which is explained in Section $\llbracket$ and illustrated in Figure 3 of the main text.

The discretisation of $\partial_{x}^{2}$ needs so-called ghost points on the fine grid, which are filled by prolongation. Our third order polynomial spatial prolongation operator is given by

$$
P_{S}[F](x):= \begin{cases}F(x) & \text { when aligned } \\
\frac{1}{16}[-1,+9,+9,-1] & \\
\cdot F(x+[-3,-1,+1,+3] \Delta x) & \text { otherwise }\end{cases}
$$

and the second order polynomial temporal prolongation operator by

$$
P_{T}[F](x):= \begin{cases}F(t) & \text { when aligned } \\
\frac{1}{8}[-1,+6,+3] & \\
\cdot F(t+[-3,-1,+1] \Delta t) & \text { otherwise }\end{cases}
$$

where $[\cdots]$ denotes a vector, and the operator $(\cdot)$ a dot product.

Let us now consider a time evolution of the above solution A11 A12. According to the Berger-Oliger time stepping scheme, the coarse grid evolution happens as on a uniform grid. The fine grid can now be evolved in two different ways: (a) with a Dirichlet boundary condition from prolongating from the coarse grid, or (b) without a boundary condition, i.e. only as an IVP (as opposed to an IBVP). In case (b), the "lost" points have to be filled by prolongation after the time step; in that case, prolongation therefore has to fill not 1 , but $k+1$ grid points for an ICN integration with $k$ iterations. This is illustrated in Figure 4 of the main text.

\section{ICN integration with prolongation}

Let us examine case (a) in more detail, in which case the fine grid boundary values at $x<0$ are given through prolongation. For the solution A11, A12, time integration and prolongation are exact, hence it is for $x<0$

$$
\begin{aligned}
& U^{(k)}\left(t_{0}+\Delta t, x\right)=\frac{1}{2}\left(t_{0}+\Delta t\right)^{2}+\frac{1}{2} x^{2} \\
& V^{(k)}\left(t_{0}+\Delta t, x\right)=t_{0}+\Delta t
\end{aligned}
$$

for all ICN iterations $k$. (This assumes that all ICN iterations end up the final time. There are also different ways of expressing ICN.)

The Euler step then leads to, for $x>0$,

$$
\begin{aligned}
& U^{(0)}\left(t_{0}+\Delta t, x\right)=\frac{1}{2} t_{0}^{2}+\frac{1}{2} x^{2}+\Delta t t_{0} \\
& V^{(0)}\left(t_{0}+\Delta t, x\right)=t_{0}+\Delta t
\end{aligned}
$$

as in A15 A18 above. For all values of $x$, this can be written as

$$
\begin{aligned}
& U^{(0)}\left(t_{0}+\Delta t, x\right)=\frac{1}{2}\left(t_{0}+\Delta t\right)^{2}+\frac{1}{2} x^{2}-\Theta(x>0) \frac{1}{2}(\Delta x)^{2} \\
& V^{(0)}\left(t_{0}+\Delta t, x\right)=t_{0}+\Delta t
\end{aligned}
$$

by using the $\Theta$ function, which is defined as

$$
\Theta(L)= \begin{cases}0 & \text { when } L \text { is false } \\ 1 & \text { when } L \text { is true. }\end{cases}
$$


The first ICN iteration leads to

$$
\begin{aligned}
& U^{(1)}\left(t_{0}+\Delta t, x\right)=\frac{1}{2}\left(t_{0}+\Delta t\right)^{2}+\frac{1}{2} x^{2} \\
& V^{(1)}\left(t_{0}+\Delta t, x\right)=t_{0}+\Delta t+\Theta(0<x<H) \frac{1}{2} \alpha^{2} \Delta t
\end{aligned}
$$

On a uniform grid, and for our solution $u$, the first ICN iteration is already exact. In general, the first ICN iteration should lead to an error which is $O\left(h^{2}\right)$. This is here not the case; clearly $V^{(1)}$ has an $O(h)$ error. However, the error is confined to a region of length $\Delta x$, so an integral norm of the error can be $O\left(h^{2}\right)$; it is not clear what this means in practice.

The result of the second ICN iteration is

$$
\begin{aligned}
& U^{(2)}\left(t_{0}+\Delta t, x\right)=\frac{1}{2}\left(t_{0}+\Delta t\right)^{2}+\frac{1}{2} x^{2}+\Theta(0<x<H) \frac{1}{4} \alpha^{2}(\Delta t)^{2} \\
& V^{(2)}\left(t_{0}+\Delta t, x\right)=t_{0}+\Delta t .
\end{aligned}
$$

This expression is the final result of the first fine grid time step. It has an error that is $O\left(h^{2}\right)$, or $O\left(h^{3}\right)$ in an integral norm. As noted above a local error that is $O\left(h^{2}\right)$ will lead to a global error of $O(h)$ when considered at a fixed time. That is, the method is only first order convergent.

A third ICN step — that we don't perform, but it would be possible to do so - would result in

$$
\begin{aligned}
U^{(3)}\left(t_{0}+\Delta t, x\right)= & \frac{1}{2}\left(t_{0}+\Delta t\right)^{2}+\frac{1}{2} x^{2} \\
V^{(3)}\left(t_{0}+2 \Delta t, x\right)= & t_{0}+\Delta t \\
& +[-2 \Theta(0<x<\Delta x)+\Theta(\Delta x<x<2 \Delta x)] \frac{1}{8} \alpha^{4} \Delta t
\end{aligned}
$$

which is worse than the result of the previous iteration. The error is again $O(h)$, but has now "infected" two grid points. The error is smaller by a factor $\alpha^{2}$, but the error at the two grid points has a different sign, indicating the start of an oscillation.

It should be noted that using higher order derivatives, e.g. using a five-point stencil for a fourth-order second derivative, does not remove this error. Similarly, using a higher order prolongation operator does not help. (We assume that it would be possible to adapt the prolongation scheme directly to the time integration scheme and arrive at a consistent discretisation in this way. We do not think that this is worthwhile in practice.)

The main problem seems to be caused by taking a second derivative, which has formally an $O(1)$ error. The usual arguments why the error should be smaller in practice [56] do not hold near the discontinuity that is introduced at the refinement boundary.

The result of simulating case (a) is shown in Figure 7 of the main text. For high resolutions, convergence seems to be only of first order. Figure 8 shows case (b), which shows second order convergence for all resolutions.

\section{The general case}

In the general case the coarse grid evolution and the prolongation will not provide exact data at the boundary on the fine grid. Instead we argue heuristically as follows. The initial data $U^{(0)}, V^{(0)}$ will be correct to order $h^{2}$,

$$
U^{n}=U\left(t^{n}, x\right)+h^{2} e^{n} f^{n}(x)+O\left(h^{3}\right)
$$

where $e^{n}$ is a constant and $f^{n}$ a smooth function of $x$. This data will be evolved forward on the coarse grid using ICN to time $t^{n+1}$ where it will also be correct to order $h^{2}$,

$$
U^{n+1}=U\left(t^{n+1}, x\right)+h^{2} e^{n+1} f^{n+1}(x)+O\left(h^{3}\right)
$$

The fine grid data will then be evolved. The first ICN step is an Euler step to $t^{n+1 / 2}$ (as the fine grid timestep is one half the coarse grid timestep). On the interior of the refined grid the result will be first order accurate in time and second order in space,

$$
U^{n+1 / 2}=U\left(t^{n+1 / 2}, x\right)+\left(\Delta t e_{t}^{n+1 / 2}+(\Delta t)^{2} e_{x}^{n+1 / 2}\right) f^{n+1 / 2}(x)+O\left(h^{3}\right) .
$$


The boundary data for the fine grid is then found by prolongation. Interpolation in space and time, assumed to be second order accurate, gives

$$
U^{n+1 / 2}(x<0)=U\left(t^{n+1 / 2}, x\right)+h^{2} e_{p}^{n+1 / 2} f^{n+1 / 2}(x)+O\left(h^{3}\right) .
$$

Therefore at the refinement boundary there is a discontinuous jump in the function,

$$
\left[U^{n+1 / 2}\right]_{x=0-}^{x=0+}=\left(h e_{t}^{n+1 / 2}+(\Delta t)^{2}\left(e_{x}^{n+1 / 2}-e_{p}^{n+1 / 2}\right)\right) f^{n+1 / 2}(x) .
$$

As shown in the case of simple polynomial data in appendix A3 the next step in the ICN loop will lead to a second order local error when the second derivative of the function is taken at the discontinuity. Thus the error at a fixed time will only be first order convergent. It is clear that this discontinuity will also lead to first order errors with other multi-step time integration methods such as the Runge-Kutta methods.

We have performed a symbolic calculation of two complete coarse grid time steps with generic initial data using Maple [57]. We find that the errors in $u$ and $v$ scale with $\Delta x^{2}$ and $\Delta x$, respectively, both after the first and the second coarse grid steps. This is consistent with the calculation above as well as our numerical results. This means that, after a fixed time $t$, the error in $u$ is $O(\Delta x)$, so that we expect first order convergence only.

[1] M. J. Berger and J. Oliger, J. Comput. Phys. 53, 484 (1984).

[2] M. Berger and P. Colella, J. Comp. Phys. 53, 64 (1989).

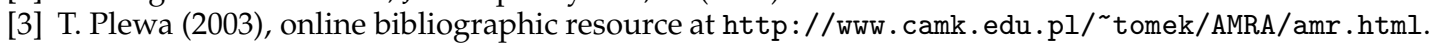

[4] K. H. A. Winkler, M. L. Norman, and D. Mihalas, J. Quant. Spec. Rad. Trans. 31, 473 (1984).

[5] K. H. A. Winkler, M. L. Norman, and D. Mihalas, J. Quant. Spec. Rad. Trans. 31, 479 (1984).

[6] T. Plewa and E. Mueller, Comp. Phys. Comm. 138, 101 (2001).

[7] A. M. Khokhlov, J. Comput. Phys. 143, 519 (1998).

[8] M. W. Choptuik, in Frontiers in Numerical Relativity, edited by C. Evans, L. Finn, and D. Hobill (Cambridge University Press, Cambridge, England, 1989).

[9] M. W. Choptuik, Phys. Rev. Lett. 70, 9 (1993).

[10] D.-I. Choi, Ph.D. thesis, University of Texas at Austin (1998).

[11] S. H. Hawley, Ph.D. thesis, University of Texas at Austin (2000).

[12] G. Lanfermann, Master's thesis, Freie Universität Berlin, MPI für Gravitationsphysik (1999).

[13] D.-I. Choi, J. D. Brown, B. Imbiriba, J. Centrella, and P. MacNeice (2003), submitted to J. Comput. Phys., physics/0307036.

[14] P. Papadopoulos, E. Seidel, and L. Wild, Phys. Rev. D 58, 084002 (1998), gr-qc/9802069.

[15] K. C. New, D.-I. Choi, J. M. Centrella, P. MacNeice, M. Huq, and K. Olson, Phys. Rev. D 62, 084039 (2000).

[16] S. L. Liebling, Phys. Rev. D 66, 041703 (2002), gr-qc/0202093.

[17] F. Pretorius, Ph.D. thesis, University of British Columbia (2002).

[18] M. W. Choptuik, E. W. Hirschmann, S. L. Liebling, and F. Pretorius, Phys. Rev. D 68, 044007 (2003), gr-qc/0305003.

[19] P. Diener, N. Jansen, A. Khokhlov, and I. Novikov, Class. Quantum Grav. 17, 435 (2000), gr-qc/9905079.

[20] L. Lowe and D. Brown (2003), talk given at Am. Phys. Soc. meeting, April 8.

[21] S. D. Hern, Ph.D. thesis, Cambridge University (1999), gr-qc/0004036.

[22] B. Brügmann, Phys. Rev. D 54, 7361 (1996).

[23] B. Imbiriba, D.-I. Choi, J. D. Brown, J. Baker, and J. Centrella (2003), talk given at Am. Phys. Soc. meeting, April 7.

[24] F. Pretorius and L. Lehner (2003), gr-qc/0302003.

[25] B. Brügmann, Int. J. Mod. Phys. D 8, 85 (1999), gr-qc/9708035.

[26] L. E. Kidder and L. S. Finn, Phys. Rev. D 62, 084026 (2000), gr-qc/9911014.

[27] L. E. Kidder, M. A. Scheel, S. A. Teukolsky, E. D. Carlson, and G. B. Cook, Phys. Rev. D 62, 084032 (2000), gr-qc/0005056.

[28] H. P. Pfeiffer, L. E. Kidder, M. A. Scheel, and S. A. Teukolsky, Comput. Phys. Commun. 152, 253 (2003), gr-qc/0202096.

[29] M. A. Scheel, L. E. Kidder, L. Lindblom, H. P. Pfeiffer, and S. A. Teukolsky, Phys. Rev. D 66, 124005 (2002), gr-qc/0209115.

[30] M. A. Scheel, A. L. Erickcek, L. M. Burko, L. E. Kidder, H. P. Pfeiffer, and S. A. Teukolsky (2003), gr-qc/0305027.

[31] G. Allen, W. Benger, T. Dramlitsch, T. Goodale, H.-C. Hege, G. Lanfermann, A. Merzky, T. Radke, and E. Seidel, in Euro-Par 2001: Parallel Processing, Proceedings of the 7th International Euro-Par Conference, edited by R. Sakellariou, J. Keane, J. Gurd, and L. Freeman (Springer, 2001).

[32] G. Allen, W. Benger, T. Goodale, H.-C. Hege, G. Lanfermann, A. Merzky, T. Radke, E. Seidel, and J. Shalf, Cluster Computing 4, 179 (2001).

[33] B. Talbot, S. Zhou, and G. Higgins (2000), http://sdcd.gsfc.nasa.gov/ESS/esmf_tasc/Files/Cactus_b.html.

[34] The Cactus Team, The Cactus computational toolkit, http://www . cactuscode .org/.

[35] T. Goodale, G. Allen, G. Lanfermann, J. Massó, T. Radke, E. Seidel, and J. Shalf, in Vector and Parallel Processing - VECPAR'2002, 5th International Conference, Lecture Notes in Computer Science (Springer, Berlin, 2002).

[36] M. W. Choptuik (1995), http://wwwrel.ph.utexas.edu/Members/matt/Doc/texas95.amr.ps. 
[37] J. Bell, M. Berger, J. Saltzman, and M. Welcome, SIAM J. Sci. Comput. 15, 127 (1994).

[38] M. J. Berger and I. Rigoutsos, IEEE Trans. on Systems, Man and Cybernetics 21, 1278 (1991).

[39] J. Quirk, Ph.D. thesis, Cranfield University (1991).

[40] M. Alcubierre, B. Brügmann, P. Diener, M. Koppitz, D. Pollney, E. Seidel, and R. Takahashi, Phys. Rev. D 67, 084023 (2003), gr-qc/0206072.

[41] M. Shibata and T. Nakamura, Phys. Rev. D 52, 5428 (1995).

[42] T. W. Baumgarte and S. L. Shapiro, Phys. Rev. D 59, 024007 (1999), gr-qc/9810065.

[43] J. York, in Sources of Gravitational Radiation, edited by L. Smarr (Cambridge University Press, Cambridge, England, 1979).

[44] C. Bona, J. Masso, E. Seidel, and J. Stela, Phys. Rev. Lett. 75, 600 (1995), gr-qc/9412071.

[45] M. Alcubierre and B. Brügmann, Phys. Rev. D 63, 104006 (2001), gr-qc/0008067.

[46] M. Alcubierre, B. Brügmann, D. Pollney, E. Seidel, and R. Takahashi, Phys. Rev. D 64, 061501 (R) (2001), gr-qc/0104020.

[47] H. Kreiss and J. Oliger, Methods for the Approximate Solution of Time Dependent Problems, vol. 10 of Global Atmospheric Research Programme (GARP): GARP Publication Series (GARP Publication, 1973).

[48] B. Brügmann (Oct. 2001), personal communication.

[49] S. Brandt and B. Brügmann, Phys. Rev. Lett. 78, 3606 (1997).

[50] B. Szilágyi, R. Gómez, N. T. Bishop, and J. Winicour, Phys. Rev. D 62, 104006 (2000), gr-qc/9912030.

[51] B. Szilágyi, B. Schmidt, and J. Winicour, Phys. Rev. D 65, 064015 (2002), gr-qc/0106026.

[52] M. Alcubierre, G. Allen, C. Bona, D. Fiske, T. Goodale, F. S. Guzmán, I. Hawke, S. Hawley, S. Husa, M. Koppitz, et al. (2003), submitted to Class. Quantum Grav., gr-qc/0305023.

[53] L. Kidder, M. Scheel, S. Teukolsky, and G. Cook, in Miniprogram on Colliding Black Holes: Mathematical Issues in Numerical Relativity (2000), http://doug-pc.itp.ucsb.edu/online/numrel00/scheel/.

[54] G. Calabrese, L. Lehner, D. Neilsen, J. Pullin, O. Reula, O. Sarbach, and M. Tiglio (2003), gr-qc/0302072.

[55] P. MacNeice, K. M. Olson, C. Mobarry, R. deFainchtein, and C. Packer, Comp. Phys. Comm. 126, 330 (2000).

[56] M. W. Choptuik, Phys. Rev. D 44, 3124 (1991).

[57] Maple, Maple, Birkhauser, 175 Fifth Ave, New York, NY 10010. 